\title{
Lowpass Filter with Wide Stopband Based on T-Shaped Resonator Loaded Step-Impedance Hairpin Resonator
}

\author{
Zhong Lunjie, Zuo Qiliang, Xu Jinping* \\ State Key Laboratory of Millimeter Waves, Southeast University, Nanjing, China \\ Email address: \\ 220170647@seu.edu.cn (Zhong Lunjie),220160639@seu.edu.cn (Zuo Qiliang),jpxu@seu.edu.cn (Xu Jinping) \\ ${ }^{*}$ Corresponding author
}

To cite this article:

Zhong Lunjie, Zuo Qiliang, Xu Jinping. Lowpass Filter with Wide Stopband Based on T-Shaped Resonator Loaded Step-Impedance Hairpin Resonator. Science Discovery. Vol. 7, No. 1, 2019, pp. 45-49. doi: 10.11648/j.sd.20190701.20

Received: April 19, 2019; Accepted: May 20, 2019; Published: May 23, 2019

\begin{abstract}
A lowpass filter with wide stopband based on stepped impedance hairpin resonator is proposed. Multiple transmission zeros are created in the stopband region by different size T-shaped resonator to achieve wide stopband. A lowpass filter with $3 \mathrm{~dB}$ cutoff frequency $\left(f_{c}\right)$ at $3.2 \mathrm{GHz}$ is designed, simulated and fabricated. Experimental results indicate that stopband can be extended to more than $7 f_{c}$ with a rejection level of $20 \mathrm{~dB}$ after loading T-shaped resonator. The insertion loss is lower than $0.4 \mathrm{~dB}$ and return loss is better than $16 \mathrm{~dB}$ in the passband. Excluding the feed lines, the size of the filter is less than $0.26 \lambda_{\mathrm{g}} \mathrm{X}$ $0.06 \lambda_{\mathrm{g}}$. The modified filter is of good value in engineering application for its small size, sharp cutoff frequency response and wide stopband.
\end{abstract}

Keywords: Hairpin Resonator, T-shaped Resonator, Lowpass Filter, Wide Stopband

\section{加载T形单元的阶梯阻抗发卡谐振器宽阻带低通滤波器}

钟伦杰, 左琪良, 徐金平*

东南大学毫米波国家重点实验室, 南京, 中国

\section{邮箱}

220170647@seu.edu.cn（钟伦杰），220160639@seu.edu.cn（左琪良）,jpxu@seu.edu.cn（徐金平）

摘要: 本文提出了一种基于阶梯阻抗发卡谐振器的宽阻带低通滤波器。通过加载不同尺寸T形谐振单元, 在阻带内引 入了多个传输零点, 从而实现滤波器的宽阻带范围。仿真设计并加工制作了 $3 \mathrm{~dB}$ 截止频率 $\left(f_{c}\right)$ 为 $3.2 \mathrm{GHz}$ 的低通滤波器。 实测结果表明, 加载 T形谐振单元后滤波器的阻带范围延拓至 $7 f_{c}$ 以上, 抑制度优于 $20 \mathrm{~dB}$, 通带内的插入损耗小于 $0.4 \mathrm{~dB}$, 回波损耗优于 $16 \mathrm{~dB}$ 。不考虑馈线长度, 整个滤波器的尺寸小于 $0.26 \lambda_{\mathrm{g}} \times 0.06 \lambda_{\mathrm{g}}$ 。改进后的滤波器体积小, 截止特性陡峭, 阻带宽, 具有较高的工程实用价值。

关键词：发卡谐振器, T形谐振单元, 低通滤波器, 宽阻带

\section{1. 引言}

无线通信系统的广泛应用是当今信息化社会发展 的重要基础。在常见的超外差接收机系统中, 接收到的
高频信号通常会经过多次变频变换到中频进行数字信 号处理, 一个性能优异, 体积小的低通滤波器能够很好 的抑制来自高频信号的干扰, 保证中频信号的质量, 提 高接收机的灵敏度。已经有众多学者进行了低通滤波器 
的研究 [1-9]。常用的低通滤波器有阶跃阻抗滤波器和短 截线低通滤波器, 但是它们都存在体积较大, 截止特性 不陡峭等缺点，因此限制了它们在射频系统中的应用。 Lung-Hua Hsieh 等人提出了一种新颖的阶梯阻抗发卡 谐振器低通滤波器结构[2]。该滤波器尺寸小, 容易制 造, 同时具有陡峭的截止频率响应特性, 但是滤波器的 阻带较窄。工程中常常通过增加谐振器的阶数来拓宽发 卡型低通滤波器的阻带[5-6], 这种方法会显著增加插入 损耗和滤波器体积。部分低通滤波器通过增加谐振单元 可以拓宽滤波器阻带 [7-9], 但从结果看出阻带增加有限 仍无法满足宽阻带的工程要求。因此研究宽阻带的高性 能低通滤波器具有重要意义。

本文在阶梯阻抗发卡低通滤波器的结构基础上,增 加了 $\mathrm{T}$ 形谐振单元, 优化谐振单元位置, 引入带外传 输零点, 极大拓展了滤波器的阻带, 同时对滤波器体积 和截止特性影响较小，具有工程实用价值。

\section{2. 基于阶梯阻抗发卡谐振器的低通滤波器}

阶梯阻抗发卡谐振器结构简单, 原理清晰, 调整方 便，通过选择不同材料的基片可以工作在不同的射频频 率范围内, 因此在实际工程中被广泛的应用于微带低通 滤波器、带通滤波器[10]、振荡器和混频器中[11]。

\section{1. 阶梯阻抗发卡谐振单元的谐振条件分析}

阶梯阻抗发卡型谐振器的结构如图 1 所示, 图 2 同时 显示其等效电路[7], 这种谐振器可以将其视作单一传输线 和终端开路的平行耦合线并联来分析。

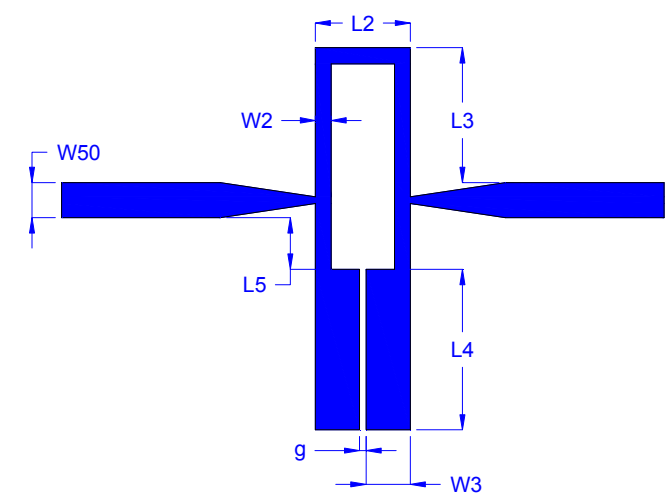

图1 阶梯阻抗发卡谐振器的结构。

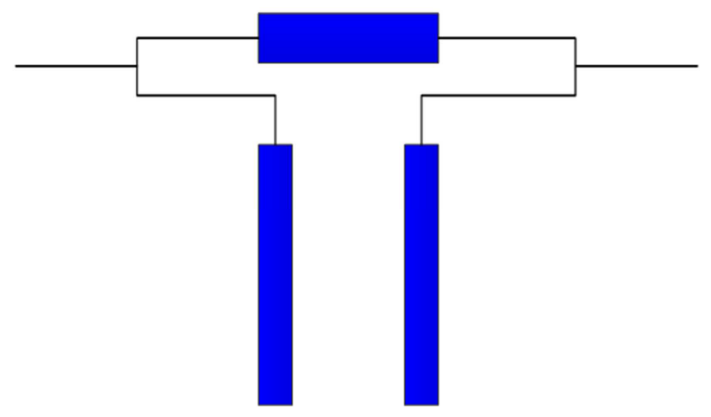

图2 阶梯阻抗发卡谐振器的等效电路。
平行耦合线的奇偶模阻抗和对应的耦合的角分别为 $Z_{0 o} 、 Z_{0 e}$ 和 $\theta_{o} 、 \theta_{e}$, 传输矩阵为:

$$
\begin{aligned}
{[F 1] } & =\left[\begin{array}{ll}
A_{1} & B_{1} \\
C_{1} & D_{1}
\end{array}\right] \\
& =\left[\begin{array}{ll}
\frac{Z_{0 e} \cot \theta_{e}+Z_{0 o} \cot \theta_{o}}{Z_{0 e} \cot \theta_{e}-Z_{0 o} \cot \theta_{o}} & -j \frac{2 Z_{0 e} Z_{0 o} \cot \theta_{e} \cot \theta_{o}}{Z_{0 o} \cot \theta_{e}-Z_{0 o} \cot \theta_{o}} \\
j \frac{2}{Z_{0 e} \cot \theta_{e}-Z_{0 o} \cot \theta_{o}} & \frac{Z_{0 o} \cot \theta_{e}+Z_{0 o} \cot \theta_{o}}{Z_{0 o} \cot \theta_{e}-Z_{0 o} \cot \theta_{o}}
\end{array}\right]
\end{aligned}
$$

单一传输线的传输矩阵为:

$$
\left[F_{2}\right]=\left[\begin{array}{ll}
A_{2} & B_{2} \\
C_{2} & D_{2}
\end{array}\right]=\left[\begin{array}{cc}
\cos \theta_{T} & j Z_{S} \sin \theta_{T} \\
j \sin \theta_{T} / Z_{S} & \cos \theta_{T}
\end{array}\right]
$$

根据传输矩阵的并联性质得到总的传输矩阵:

$$
\begin{gathered}
{\left[F_{T}\right]=\left[\begin{array}{ll}
A_{T} & B_{T} \\
C_{T} & D_{T}
\end{array}\right]} \\
A_{T}=\frac{A_{1} B_{2}+A_{2} B_{1}}{B_{1}+B_{2}}, B_{T}=\frac{B_{1} B_{2}}{B_{1}+B_{2}} \\
C_{T}=\frac{-\left(A_{1}-A_{2}\right)\left(D_{1}-D_{2}\right)-\left(B_{1}+B_{2}\right)\left(C_{1}+C_{2}\right)}{B_{1}+B_{2}} \\
D_{T}=\frac{B_{1} D_{2}+B_{2} D_{1}}{B_{1}+B_{2}}=A_{T}
\end{gathered}
$$

由总的传输矩阵得到负载为 $Z_{L}$ 时的输入导纳

$$
Y_{i}=\frac{C_{T} Z_{L}+D_{T}}{A_{T} Z_{L}+B_{T}}
$$

考虑 $Z_{L}$ 无穷大, $\theta_{o}=\theta_{e}=\theta_{0}$, 得到谐振条件[11]

$$
\begin{aligned}
& \left(Z_{0 e} \cdot Z_{0 o} \cot \theta_{0}-Z_{S}^{2} \tan \theta_{0}\right) \sin 2 \theta_{s}+ \\
& Z_{S}\left(Z_{e}+Z_{o}\right) \cos 2 \theta_{s}-Z_{S}\left(Z_{e}+Z_{o}\right)=0
\end{aligned}
$$

式中 $\theta_{S}=\theta_{T} / 2$, 定义 $Z_{0}=\sqrt{Z_{0 e} Z_{0 o}}$, $C=\left(Z_{0 o}-Z_{0 e}\right) /\left(Z_{0 o}+Z_{0 e}\right), R_{Z}=Z_{P} / Z_{S}$ 可得到进一步简 化的谐振条件:

$$
\begin{aligned}
& \sqrt{1-C^{2}} \cdot\left(R_{Z} \cot \theta_{0}-\tan \theta_{0} / R_{Z}\right) \cdot \sin 2 \theta_{s}+ \\
& 2 \cos 2 \theta_{S}+2 \cos 2 \theta_{S}-2 C=0
\end{aligned}
$$

通过以上结果, 可以得到谐振器总长度和单线长度 的关系，用以讨论小型化条件。减小 $R_{Z}$ 或增大 $\mathrm{C}$ 可以 使谐振器尺寸变小。

\section{2. 低通滤波器仿真}

基于以上理论, 本文设计了一款低通滤波器, 具体 
指标为: 通带 $0-2.2 \mathrm{GHz}$, 插入损耗小于 $1 \mathrm{~dB}$, 回波损 耗优于 $15 \mathrm{~dB}$, 在 $4.2 \mathrm{GHz}$ 处抑制大于 $20 \mathrm{~dB}$ 。采用 Rogers $4350 \mathrm{~B}$ 作为基板材料, 厚度 $\mathrm{H}=0.508 \mathrm{~mm}, \varepsilon_{r}=3.66$, 铜厚 $\mathrm{T}=0.018 \mathrm{~mm}$, 在 HFSS 中建模仿真并优化, 得到 一组 结构参数: $\mathrm{L} 2=2 \mathrm{~mm}, \mathrm{~L} 3=3.7 \mathrm{~mm}, \mathrm{~L} 4=5 \mathrm{~mm}$, $\mathrm{L} 5=0.8 \mathrm{~mm}, \mathrm{w} 3=2.65 \mathrm{~mm}, \mathrm{~g}=0.2 \mathrm{~mm}$ 。滤波器的 $\mathrm{S}$ 参数仿 真结果如图 3 所示。

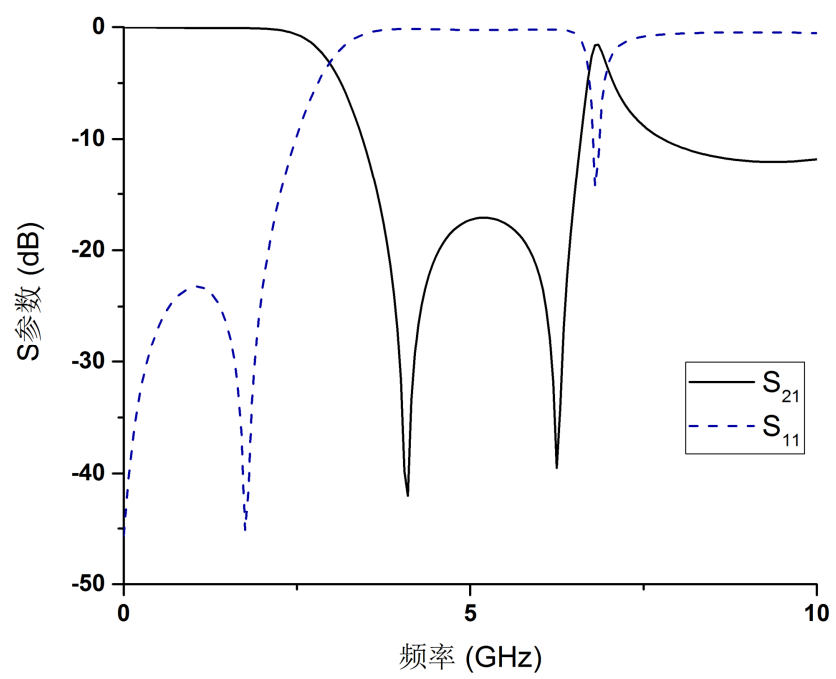

图3 传统的发卡谐振器低通滤波器仿真结果。

从上图中可以看出, 在通带 $0-2.2 \mathrm{GHz}$ 范围内, 插 入损耗小于 $0.3 \mathrm{~dB}$, 回波损耗优于 $20 \mathrm{~dB}, 3 \mathrm{~dB}$ 截止频率 为 $2.9 \mathrm{GHz}$, 在 $4.2 \mathrm{GHz}$ 处抑制大于 $29 \mathrm{~dB}$, 在 $3.8-6.5 \mathrm{GHz}$ 内抑制大于 $17 \mathrm{~dB}$ 。滤波器截止特性陡峭, 但是阻带范 围小，寄生通带多，在实际应用中对高频信号抑制不够 深, 限制了该类型滤波器的使用。因此滤波器需要进一 步改进以实现更宽的阻带。

\section{T形谐振单元分析}

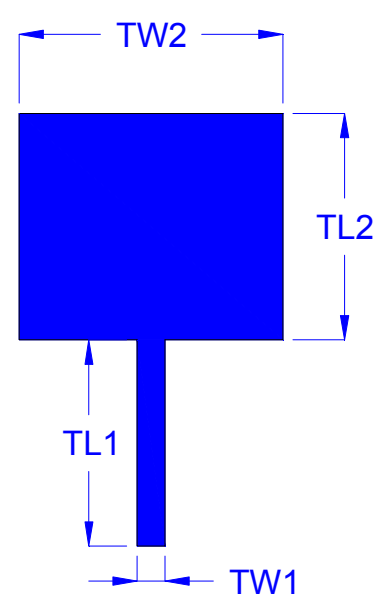

图4 T形谐振单元结构。

图 4 所示的 $\mathrm{T}$ 形单元是一种简单的四分之波长谐 振器, 在滤波器设计中得到了广泛的使用。它能以很
简单的结构引入传输零点, 提高滤波器的带外性能。可 以将高阻抗线等效为电感, 低阻抗线等效为电容来进行 简单的定性分析。整个开路枝节等效为一串联谐振电路, 当减小高阻抗线的长度（减小电感值）或减小低阻抗线 的宽度 (减小电容值) 均可使谐振频率变大, 传输零点 上移。

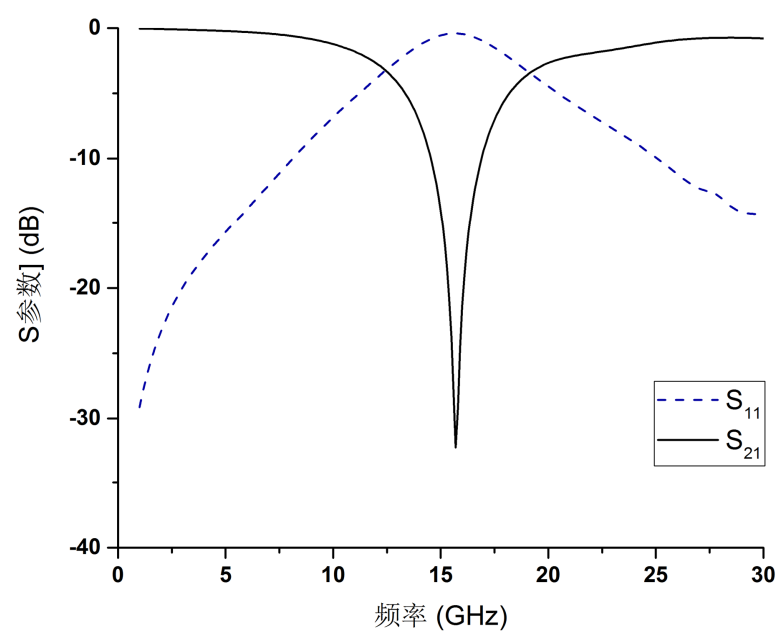

图5 T形谐振单元 $\mathrm{S}$ 参数仿真结果。

谐振频率为 $15.7 \mathrm{GHz}$, 谐振单元的长度为 $1.7 \mathrm{~mm}$, 小于谐振频率四分之一波导波长 $2.4 \mathrm{~mm}$, 这是因为谐 振器采用阶梯阻抗形式, 阻抗比小于 1 , 因此谐振器长 度比传统的四分之一波长谐振器长度要短。

改变 $\mathrm{T}$ 形谐振单元高阻抗线的长度, 分别为 $0.4 \mathrm{~mm}, 0.3 \mathrm{~mm}$, 得到的仿真结果如图 6 。

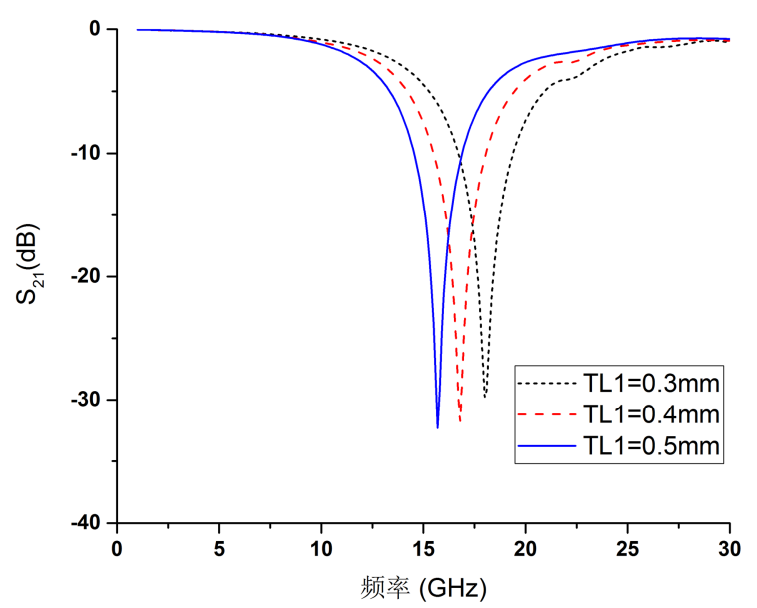

图6 不同高阻抗线长度的 $\mathrm{T}$ 形单元的 $\mathrm{S}$ 参数。

从仿真结果可以看出对应的谐振频率为 $16.8 \mathrm{GHz}$ 和 $18.0 \mathrm{GHz}$, 减少 TL1 的长度, 谐振频率上移, 这与 前面的分析是一致的。

\section{4. 阶梯阻抗发卡滤波器加载T形谐振单元}

综合以上仿真分析结果, 将三个 $\mathrm{T}$ 形单元加载在 
滤波器的不同位置, 具体结构如图 7 所示:

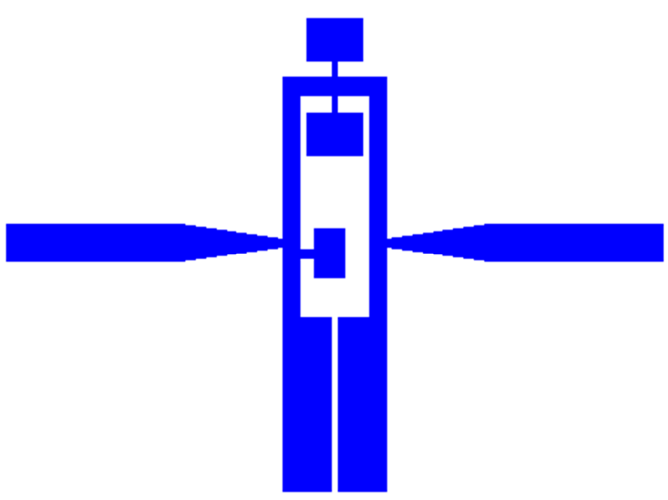

图7 加载T形谐振单元后的滤波器结构。

不同尺寸的 $\mathrm{T}$ 形单元对应不同的带外传输零点, 起到拓宽阻带的作用, 并且加载在发卡内部可以节省空 间, 减小滤波器体积。发卡滤波器的基本尺寸保持不变。 同时采用雉形渐变线馈电, 实现输入阻抗匹配, 改善驻 波, 减小反射。图 8 给出了加载三个 $\mathrm{T}$ 形单元后滤波 器的 HFSS 仿真结果。

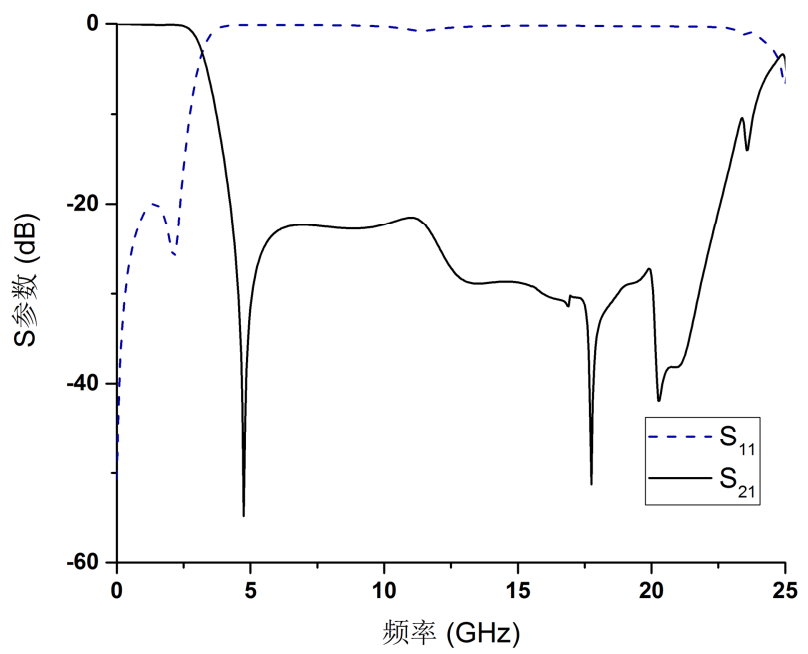

图8 加载三个 $\mathrm{T}$ 形单元后滤波器的 $\mathrm{S}$ 参数仿真结果。

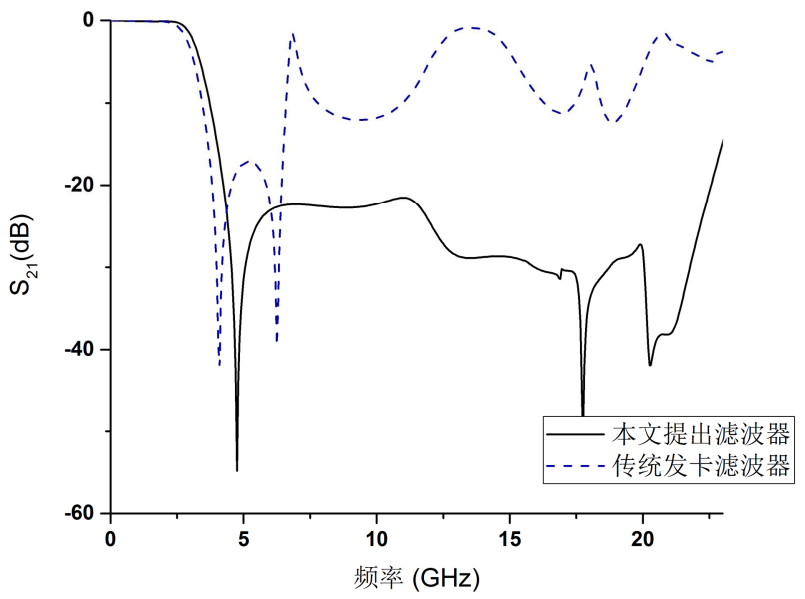

图9 改进前后滤波器 $\mathrm{S}_{21}$ 对比。
由图 9 可见, 在通带 $0-2.2 \mathrm{GHz}$ 内 $\mathrm{S} 11$ 低于 $-15 \mathrm{~dB}$, 带内插入损耗小于 $0.5 \mathrm{~dB}$, 与未加载 $\mathrm{T}$ 形单元前基本一 致, $3 \mathrm{~dB}$ 截止频率为 $3.2 \mathrm{GHz}$, 在 $4.2 \mathrm{GHz}$ 处抑制为 $19 \mathrm{~dB}$, 增加的枝节会使滤波器频带向右偏移。同时在 4.23-22.5GHz 范围内抑制大于 $20 \mathrm{~dB}$, 滤波器的阻带有 了明显拓宽。不考虑馈线长度, 整个滤波器的尺寸为 $0.26 \lambda_{\mathrm{g}} \times 0.06 \lambda_{\mathrm{g}}(12.79 \mathrm{~mm} \times 3 \mathrm{~mm})$, 其中 $\lambda_{\mathrm{g}}$ 是 $3 \mathrm{~dB}$ 截止频 率 $3.2 \mathrm{GHz}$ 对应的导波波长。

\section{5. 实验结果}

根据 HFSS 仿真的具体尺寸加工了滤波器实验样 品, 图 10 是其实物照片。利用 Agilent E8363C 型号的 矢量网络分析仪进行 $\mathrm{S}$ 参数测量, 该设备可以测量的 范围从 $10 \mathrm{MHz}$ 到 $40 \mathrm{GHz}$ 。实测结果与仿真结果的比较 如图 11 所示, 通带内插入损耗小于 $0.4 \mathrm{~dB}$, 带内回波 损耗优于 $16 \mathrm{~dB}, 3 \mathrm{~dB}$ 截止频率位于 $3.2 \mathrm{GHz}$ 。在 $4.2 \mathrm{GHz}$ 处抑制为 $17 \mathrm{~dB}$, 在 $4.3 \mathrm{GHz}-22.7 \mathrm{GHz}$ 的阻带范围内衰减 大于 $20 \mathrm{~dB}$ 。实测结果与仿真结果一致性较好。

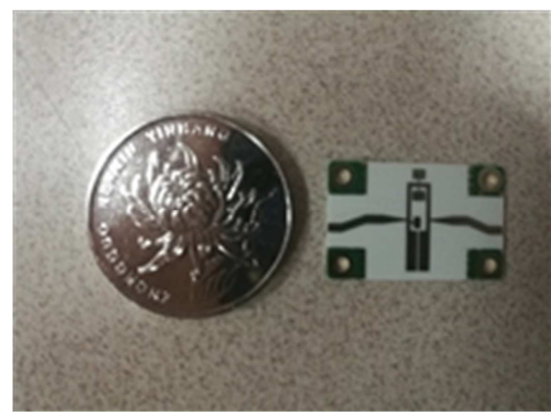

图10 滤波器加工实物图。

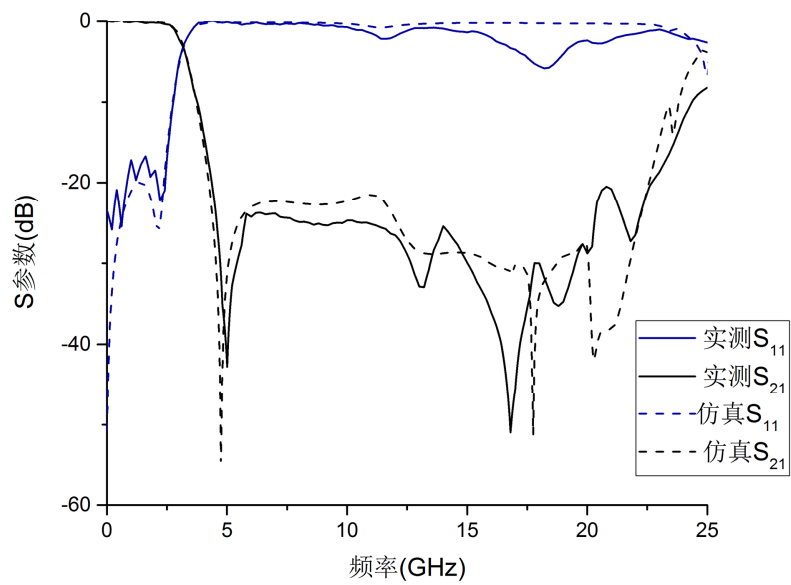

图11 滤波器仿真结果与实测结果对比。

\section{6. 结论}

本文在阶梯阻抗发卡滤波器基本结构上, 通过加载 $\mathrm{T}$ 形谐振单元, 拓宽了低通滤波器阻带。实测结果表明, 加载三个 $\mathrm{T}$ 形单元后, 阻带由 3.8-6.5 GHz 展宽到 4.3-22.5GHz, 阻带抑制度均大于 $20 \mathrm{~dB}$, 部分频点抑制 
达到 $30 \mathrm{~dB}$, 同时对滤波器截止特性影响较小。该改进 后的滤波器体积小, 截止特性陡峭, 阻带宽, 具有较高 的工程实用价值。

\section{参考文献}

[1] 蔡钟斌. 基于阶梯阻抗发夹谐振器的小型低通滤波器 $[\mathrm{J}]$. 太赫兹科学与电子信息学报, 2007, 5(1):78-80。

[2] Hsieh L H, Chang K. Compact lowpass filter using stepped impedance hairpin resonator [J]. Electronics Letters, 2001, 37 (14): 899.

[3] Jiang $\mathrm{S}, \mathrm{Xu}$ J. Compact microstrip lowpass filter with ultra-wide stopband based on dual-plane structure [J]. Electronics Letters, 2017, 53 (9): 607-609.

[4] 傅世强, 张佳琦, 房少军. 一种超宽阻带微带低通滤波器 的设计 [J]. 微波学报, 2018, 34(5):41-43。

[5] Hsieh L H, Chang K. Compact elliptic-function low-pass filters using microstrip stepped impedance hairpin resonator
[J]. IEEE Transactions on Microwave Theory and Techniques, 2003, 51 (1): 193-199.

[6] Li Z, Wang G M, Zhang C X, et al. Compact ultra-wide bandpass filter with good out-of-band performance [C] // International Conference on Microwave \& Millimeter Wave Technology. IEEE, 2008.

[7] Li R, Kim D I, Choi C M. Compact Structure With Three Attenuation Poles for Improving Stopband Characteristics [J]. IEEE Microwave and Wireless Components Letters, 2006, 16 (12): 663-665.

[8] Yang M H, Xu J. Design of compact, broad-stopband lowpass filter using modified stepped impedance hairpin resonators $[\mathrm{J}]$. Electronics Letters, 2008, 44 (20): 1198-1199.

[9] Wei X B, Wang P, Liu M Q, et al. Compact wide-stopband lowpass filter using stepped impedance hairpin resonator with radial stubs [J]. Electronics Letters, 2011, 47 (15): 862.

[10] 毛睿杰, 唐小宏, 王玲. 宽阻带阶跃阻抗发夹线带通滤波 器设计[J]. 微波学报, 2007(1):44-46。

[11] Makimoto, Mitsuo. Microwave Resonators and Filters for Wireless Communication [M]. Springer, 2010, 4: 88-89. 